\title{
Article \\ Quadruple-Mode Wideband Bandpass Filter with Improved Out-of-Band Rejection
}

\author{
Musab Hameed ${ }^{1, *(\mathbb{D}}$, Gaobiao Xiao ${ }^{1}{ }^{\mathbb{D}}$, Ali Imran Najam ${ }^{2}$, Lina Qiu ${ }^{1}$ and Tayyab Hameed ${ }^{3}$ \\ 1 Key Laboratory of Ministry of Education for Research of Design and EMC of High Speed Electronic Systems, \\ Shanghai Jiao Tong University, Shanghai 200240, China; gaobiaoxiao@sjtu.edu.cn (G.X.); \\ linaqiu@sjtu.edu.cn (L.Q.) \\ 2 National Electronics Complex of Pakistan, Islamabad 44000, Pakistan; alimranajam@gmail.com \\ 3 Department of Electrical \& Computer Engineering, Center for Advanced Studies in Engineering (CASE), \\ Islamabad 44000, Pakistan; tayyabhameed11@gmail.com \\ * Correspondence: musab88@sjtu.edu.cn
}

Received: 29 January 2019; Accepted: 4 March 2019; Published: 7 March 2019

check for updates

\begin{abstract}
This paper proposes a method for designing a quadruple-mode wideband bandpass filter using off-centered perturbed metallic cylinders in a rectangular waveguide cavity with compact size and improved out-of-band rejection. Two off-centered perturbation cylinders were placed at the bottom of the rectangular waveguide cavity along with a pair of perpendicularly-fed coaxial lines, which excited four quasi-transverse magnetic (TM) modes to realize the desired passband. The height of the waveguide cavity and the shape of the perturbation cylinders were exploited to achieve an all quasi-TM modes filter with good out-of-band rejection and sharp skirt selectivity. The proposed filter operates at $2.93 \mathrm{GHz}$ center frequency with $38 \%$ wide fractional bandwidth (FBW). The proposed filter is fabricated using aluminum. The measured and simulated results are in good agreement with each other.
\end{abstract}

Keywords: microwave filter; multi-mode resonator; quadruple-mode filter; cavity filter

\section{Introduction}

Microwave cavity filters with low insertion loss, high power handling capability, excellent frequency selectivity, and miniaturized profile are greatly desired for base stations of the advanced wireless communication systems. Previously, microwave cavity filters were implemented with single-mode cavities [1-3]. In [1,2], four and eight waveguide-coupled single-mode cavities were employed to design elliptic and narrowband filters, respectively. In [3], the filter synthesis method of identical narrowband high- $Q$ cavities was utilized to design a sixth-order elliptic narrowband filter.

On the other hand, bandpass filters (BPFs) with larger fractional bandwidth are vastly required to qualify for the advanced wireless communication systems. Until recently, the majority of waveguide cavity BPFs reported were single-mode cavity filters [4,5], but these single-mode cavity BPFs are constrained with narrow bandwidth and are larger in size. To be eligible for the base-station employments in a wireless communication system, miniaturization of the waveguide cavity filters is very much in demand. One of the methods to achieving miniaturization of cavity filters is the usage of multi-mode resonators (MMRs) [6-10]. Lin [11] was the first to design an MMR filter in 1951, after which many MMR filters were designed using several perturbation methods, such as half-cut dielectric loading [12,13], screws [14], irises [15,16], and corner-cuts [15,17]. A quadruple-mode filter based on modified coaxial cavity resonators was also designed using four coaxial structures in a single cavity [18]. All of the aforementioned works were constrained with a narrow bandwidth. 
In order to achieve wider fractional bandwidth, an off-centered metallic-loaded perturbation technique was proposed, employing a single waveguide cavity [19-21]. In [19], off-centered metallic cylinders were placed at the bottom of a waveguide cavity to separate the degenerate modes and in return realize a wideband triple or quadruple-mode filter. In [20], a mushroom-shaped perturbation cylinder was introduced along with the screw supported from the top wall instead of the conventional metal cylinder, which resulted in almost double the fractional bandwidth (FBW) with almost half the cavity size. Similarly, a special cavity-shape was utilized in [21] to design a wideband triple-mode filter. Although a wide FBW was achieved in these works, these filters were constrained with poor out-of-band rejection.

Careful investigation shows that in many designs, the three edge lengths of the cavity are very close, thus many resonant modes are crowded in the out-of-band frequency range. There always exist one or several resonant modes that strongly couple with the probes and cause poor rejection in the out-of-band. In this paper, we propose to reduce the resonant modes in the out-of-band region by largely cutting down the height of the cavity. With smaller height, those resonant modes with multiple vertical oscillations are shifted far higher away. Most of the lower resonant modes are quasi-TM mode. It is effective to adjust them with vertically-placed metal poles in the cavity to get better out-of-band rejection. To validate this idea, a quadruple-mode wideband bandpass filter was designed based on our previous works $[20,21]$, where mushroom shaped metallic perturbation cylinders were used to further reduce the cavity size. Two metallic rods were inserted into the cavity via its perpendicular sidewalls to act as input and output probes. The proposed filter targeted more optimum sets of resonant modes, which were different from [19], by exploiting the cavity dimensions. The lowest two quasi-TM modes and two additional resonant modes caused by the input/output probes contributed to passband. The quadruple-mode bandpass filter was designed at $2.93 \mathrm{GHz}$ with $38 \%$ wide FBW. A prototype filter was fabricated to vindicate the experimental results of the proposed filter. Measured results agreed well with the simulated ones.

\section{Quadruple-Mode Resonator}

\subsection{Configuration and Characteristics}

Figure 1 illustrates the physical configuration of the proposed quadruple-mode resonator, which was comprised of two mushroom-shaped conductive cylinders along with a pair of perpendicularly fed coaxial lines incorporated in a single rectangular waveguide cavity. Two screws penetrated the top wall of the cavity right above the off-centered perturbation cylinders to fine tune the coupling and resonant frequencies. The resonator was symmetric with respect to the $s-s^{\prime}$ plane. One end of the conductive cylinders was short-circuited on the bottom of the cavity, while the other end was kept open. The gap generated between the caps of the conductive cylinders and the top wall of the cavity formed a capacitor dependent on the area of the cylinders-caps and their distance from the top wall of the cavity. Increase in the capacitance caused a significant decrease in the frequencies of the resonant modes, which in turn reduced the cavity-size to retain a particular frequency [20]. The size of the rectangular waveguide cavity was described with the base length (parameter $a$ ) and the height (parameter $b$ ), which were about $\lambda_{o} / 2$ and $\lambda_{o} / 4$, respectively (where $\lambda_{o}$ refers to the wavelength taken at the center frequency). The realization of input and output ports took place by extending the inner conductor of the coaxial lines into the waveguide cavity via its perpendicular sidewalls. The probes were positioned under the caps of the off-centered mushroom-shaped cylinders. Instead of the simple conductive cylinders used in the conventional off-centered perturbed resonators [19], the mushroom-shaped conductive cylinders were used to reduce the cavity-size to less than half and improve the out-of-band rejection significantly with wider fractional bandwidth [20]. 


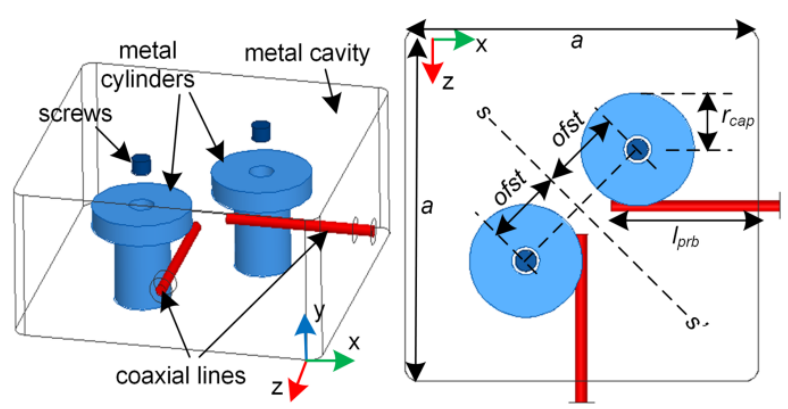

(a)

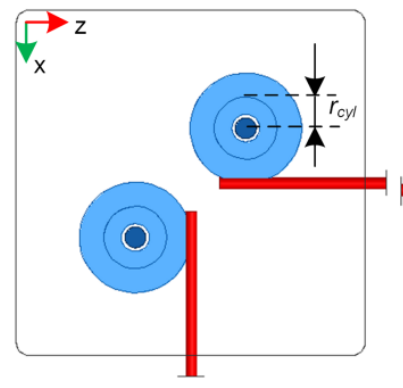

(c) (b)

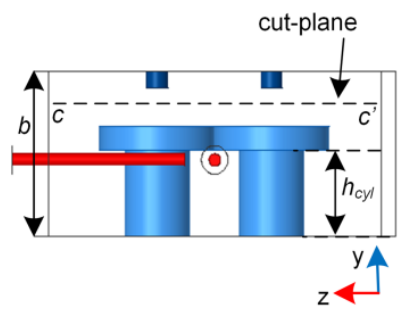

(d)

Figure 1. Structure of the proposed quadruple-mode filter (a) perspective view; (b) top view; (c) bottom view; (d) side view.

This special-shaped cylinder configuration, along with the smaller height of the cavity, revealed new resonant modes for realization of the quadruple-mode filter. The two perturbation cylinders and the two input/output probes were responsible for the excitation of the four resonant modes. The two perturbation cylinders largely contributed to the excitation of the first two resonant modes, while these cylinders (in combination with the two feeding probes) excited the remaining two modes.

By examining electromagnetic field distributions of the resonant modes in Figure 2, it was observed that all four resonant modes were quasi-TM modes. The H-field distributions of all these modes are included in the Figure to provide understanding of the resonance. The field distributions were observed from the cut-plane $c-c^{\prime}$ parallel to the xoz-plane, as is clearly graphed in Figure $1 \mathrm{~d}$. The first two resonant modes mainly affected by the two capped cylinders included quasi-TM $\mathrm{M}_{01}$ and quasi- $\mathrm{TM}_{11}$ mode, which can be seen in Figure 2a,b, respectively. Besides these, there were other two modes - namely quasi-TM mode- 3 and quasi-TM mode-4-with fields concentrating around the two probes, as clearly depicted in Figure 2c,d, respectively. 


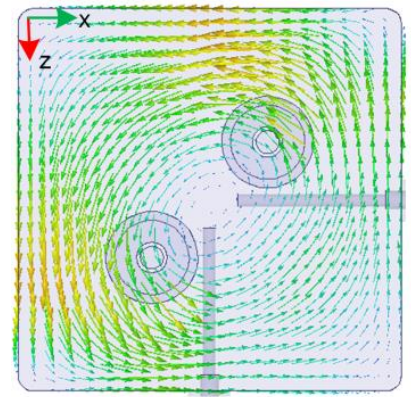

(a)

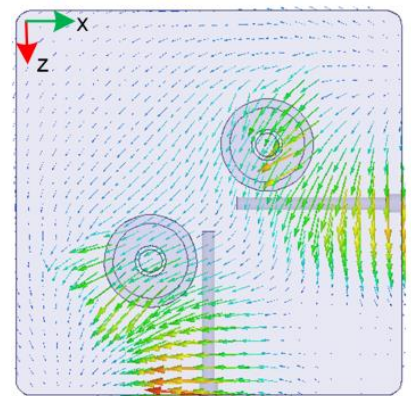

(c)

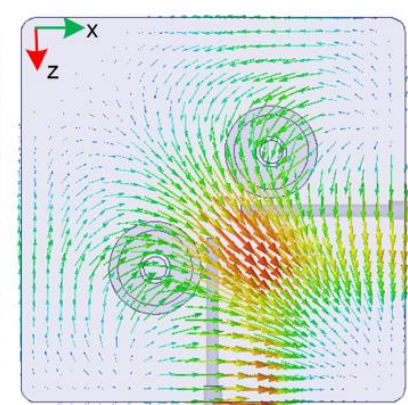

(b)

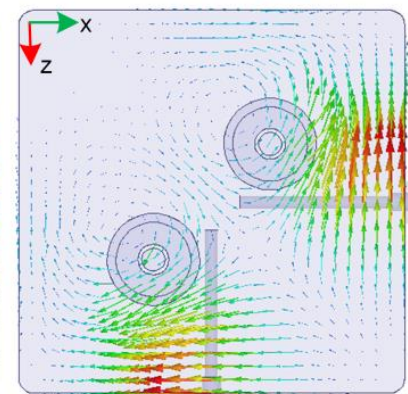

(d)

Figure 2. Field distributions of the five resonant modes: (a) magnetic field distribution of quasi-TM $\mathrm{TM}_{01}$ mode; (b) magnetic field distribution of quasi-TM $\mathrm{TM}_{11}$ mode; (c) magnetic field distribution of quasi-TM mode-3; (d) magnetic field distribution of quasi-TM mode-4.

\subsection{Parametric Optimization}

Height of the perturbation cylinders (parameter $h_{c y l}$ ), offset-shift of the perturbation cylinders from the cavity-center (parameter ofst), and length of the coupling probes (parameter $l_{p r b}$ ) were three critical parameters to control resonant frequencies of the four modes. The mode-chart comprised of resonant frequencies of the first four resonant modes along with the lowest higher-order mode graphed against these critical parameters is displayed in Figure 3. Figure 3a shows the variation in height of the perturbation cylinders (parameter $h_{c y l}$ ) from $5 \mathrm{~mm}$ to $13 \mathrm{~mm}$ while fixing ofst $=6 \mathrm{~mm}$. By increasing the value of $h_{c y l}$, the resonant frequencies of the first two modes (quasi-TM $\mathrm{TM}_{01}$, quasi-TM $\mathrm{TM}_{11}$ ) could be lowered significantly. For the lower values of $h_{c y l}$, the fourth and fifth resonant modes (mode- 4 and lowest higher-order mode) were merged into each other. As the value of the $h_{c y l}$ increased beyond $6 \mathrm{~mm}$, the two modes started separating, and mode-4 moved towards mode- 3 until an optimum value (line $x-x^{\prime}$ ) was reached. Similarly, in Figure $3 b$, the optimum value (line $y-y^{\prime}$ ) of the offset shift parameter ofst could be determined by varying its value from $6 \mathrm{~mm}$ to $8 \mathrm{~mm}$ while keeping the already optimized value of $h_{c y l}$ fixed at line $\mathrm{x}-\mathrm{x}^{\prime}$. Insertion of the two coaxial line probes lowered the resonant frequencies of mode- 3 and mode- 4 significantly and brought them into the passband to realize the quadruple-mode filter, as shown in Figure $3 c$. The length of the probes (parameter $l_{p r b}$ ) increased starting from $0 \mathrm{~mm}$ until it reached its optimum value represented by line $z-z^{\prime}$. The passband was realized by distributing all the resonant modes evenly. 


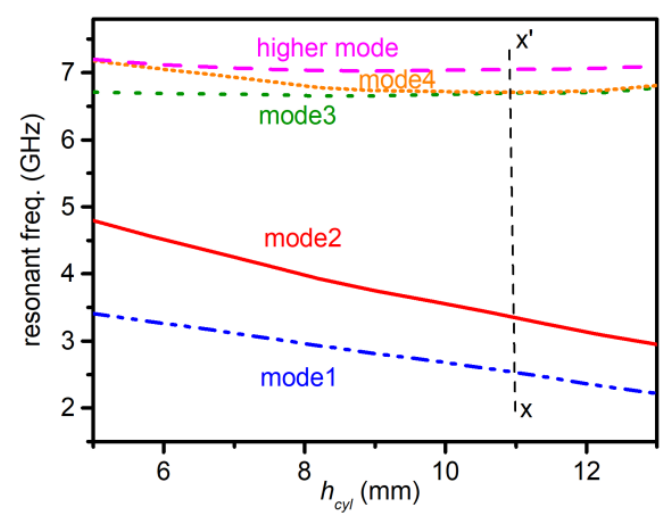

(a)

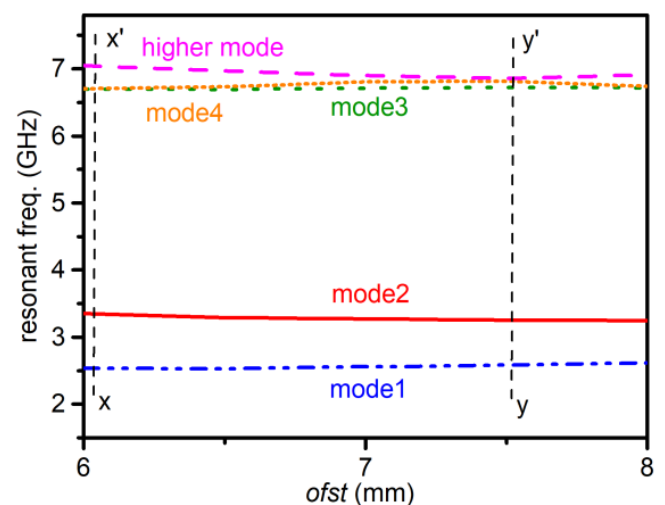

(b)

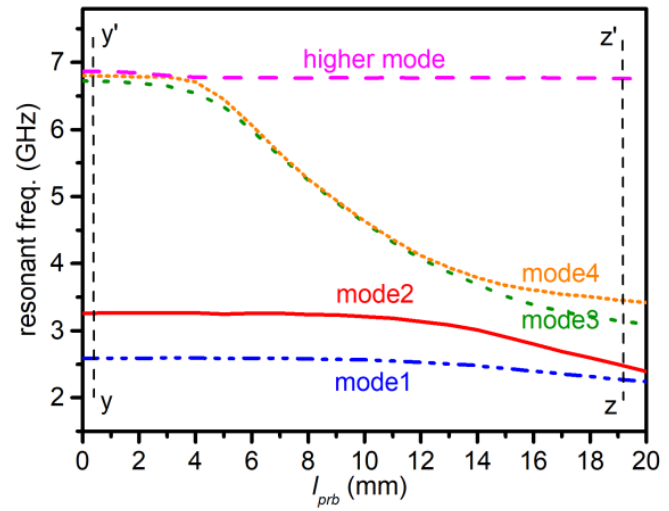

(c)

Figure 3. Mode-chart comprised of resonant frequencies of the first five resonant modes against different parameters of the filter: (a) resonant frequencies against height of the cylinders $h_{c y l}$; (b) resonant frequencies against offset shift parameter ofst; (c) resonant frequencies against length of coupling probes $l_{p r b}$.

\section{Bandpass Filter Design}

A compact quadruple-mode wideband bandpass filter was designed based on the configurations and characteristics of the proposed quadruple-mode resonator discussed in a previous section. The bandpass filter was designed with wide bandwidth, improved out-of-band rejection, high $Q$-factor, and good power handling capability. This section covers the design methodology of the proposed filter.

\subsection{Coupling Mechanism}

The transversal coupling topology was implemented to design the quadruple-mode filter [22], as depicted in Figure 4. The four shaded circles labeled as 1, 2, 3, and 4 represent the four resonant 
modes, while the two white circles on opposite sides represent the source and the load. The solid and dotted lines connecting resonators, source, and load show coupling and weak coupling among them, respectively. The mutual coupling among the four resonant modes was weak and could be neglected. The values of the coupling matrix were extracted using the extraction method explained in [23]. The coupling matrix explaining the filtering function is presented in (1).

$$
M=\left[\begin{array}{cccccc}
0 & 0.4216 & 0.6532 & 0.5969 & 0.3635 & 8.5776 \times 10^{-4} \\
0.4216 & 1.2235 & 0 & 0 & 0 & -0.4214 \\
0.6532 & 0 & 0.5804 & 0 & 0 & 0.6537 \\
0.5969 & 0 & 0 & -0.6656 & 0 & -0.5965 \\
0.3635 & 0 & 0 & 0 & -1.0104 & 0.3635 \\
8.5776 \times 10^{-4} & -0.4214 & 0.6537 & -0.5965 & 0.3635 & 0
\end{array}\right]
$$

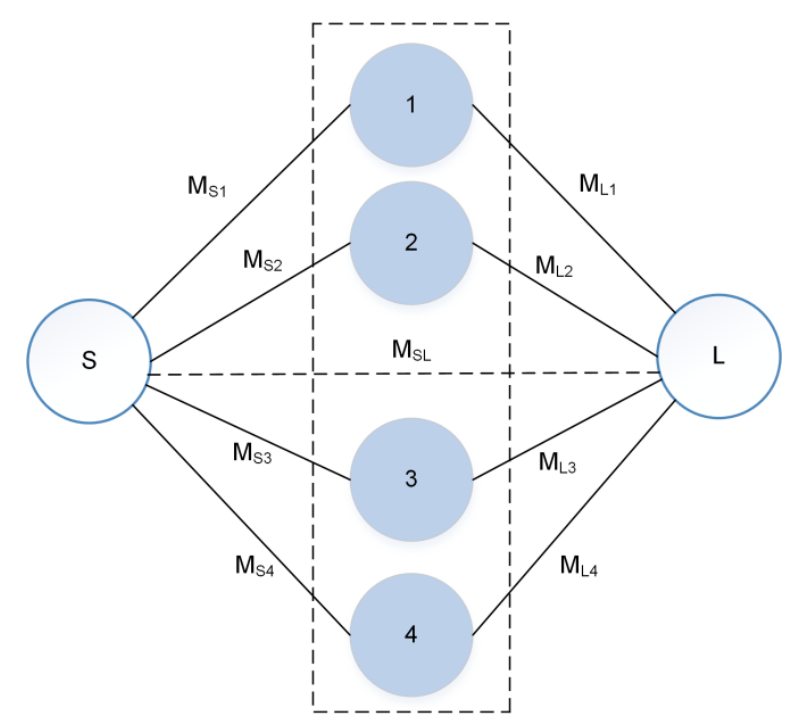

Figure 4. Coupling scheme of the quadruple-mode filter.

The filter responses acquired from HFSS simulations and the coupling matrix are compared in Figure 5 to validate the accordance of the simulation model with the coupling matrix. The results of the two models showed a good agreement between each other.

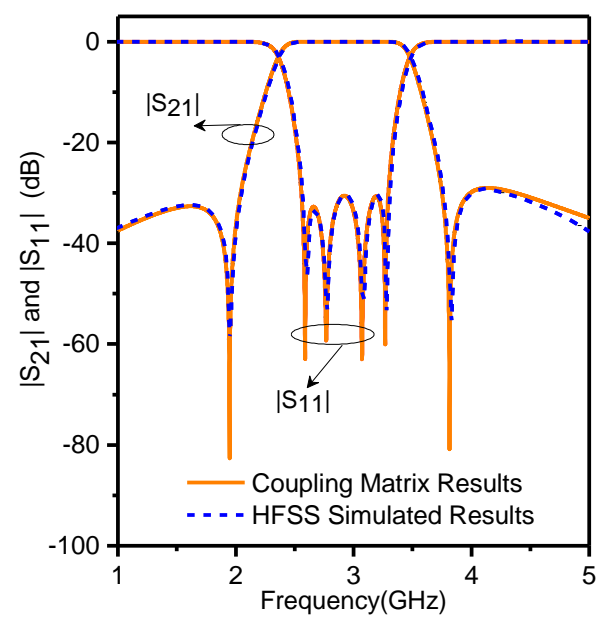

Figure 5. Simulated results (dotted) and coupling matrix results (solid) of reflection and transmission coefficients of the quadruple-mode filter. 
Two transmission zeros appeared on both sides of the passband due to the source-load coupling induced by perpendicularly placed coaxial probes.

\subsection{Q-factors}

The electromagnetic (EM) simulator Ansoft HFSS was used to obtain the values of the unloaded $Q$-factors $\left(Q_{u}\right)$ and the loaded $Q$-factors $\left(Q_{l}\right)$ while keeping the geometrical parameters of the coaxial probes the same. However, the boundary conditions applied to the two ports were Perfect $\mathrm{E}$ and matched-50 $\Omega$ for $Q_{u}$ and $Q_{l}$, respectively. The external $Q$-factor $\left(Q_{e}\right)$ for every resonant mode could be calculated by:

$$
\frac{2}{Q_{e}}=\frac{1}{Q_{l}}-\frac{1}{Q_{u}}
$$

$Q_{e}$ is generally defined for a single port, whereas in this case, the simulations to find $Q_{e}$ were carried out by setting both ports terminated with outer resisters. The loss associated with $Q_{e}$ was halved for one port, which resulted in multiplying a constant two on the left side of Equation (2).

The $Q_{e}$ of the four resonant modes graphed against $l_{p r b}$ are shown in Figure 6 . For the lower values of $l_{p r b}$, the values of $Q_{e}$ for the first two quasi-TM modes were very high, which represented their weak coupling with the input and output coupling probes. When the value of $l_{p r b}$ increased beyond $14 \mathrm{~mm}$, the $Q_{e}$ of all the four resonant modes attained stability and remained under 50 . The values of $Q_{e}$ for the four resonant modes were $Q_{e 1}=17.93, Q_{e 2}=8.27, Q_{e 3}=11.95$, and $Q_{e 4}=41.61$. The unloaded quality factors for the same modes were 5336, 3234, 4616, and 6864, respectively.

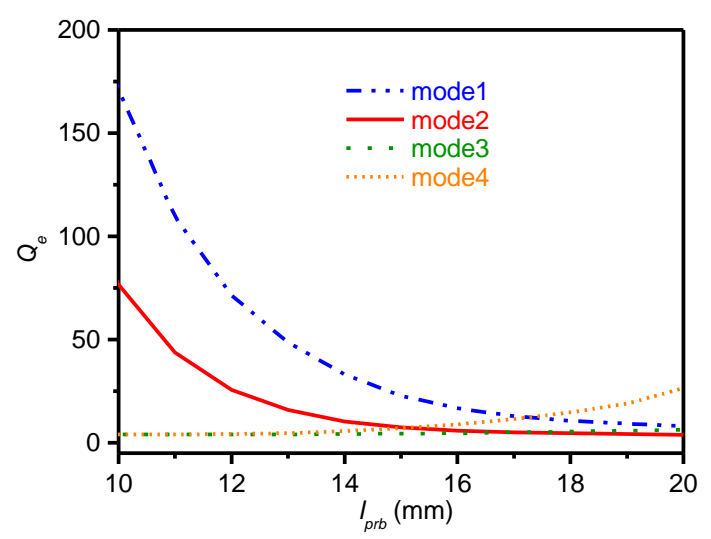

Figure 6. External quality factor $Q_{e}$ against varying $l_{p r b}$.

\subsection{Out-of-Band Rejection}

The out-of-band rejection of the filter was improved largely by exploiting the height of the waveguide cavity, $b$. The lowest higher order mode was restricted towards the higher frequencies by choosing the appropriate value of $b$, as clearly depicted in Figure 7. For $b \geq a$, the lowest higher-order mode moved very close to the passband, providing narrow out-of band rejection, while for $b<0.5 a$, the higher mode could be pushed away from the passband to achieve a wider out-of-band rejection with sharp attenuation. Figure 7 shows how a cavity size could be critical in achieving the desired results. Along with $b$, the mushroom shape of the perturbation cylinders was also crucial in lowering the resonant frequencies, which in turn reduced the overall cavity size [20]. 


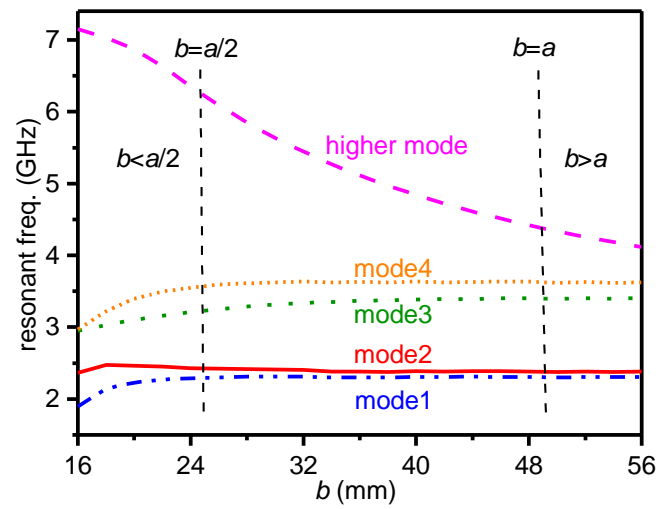

Figure 7. Resonant frequencies against height of the waveguide cavity $b$.

\subsection{Power Handling Capability}

The power handling capability of a cavity is usually measured with factor $\zeta=W / E_{\max }^{2}$, where $W$ is the total electro-magnetic energy stored in the cavity, and $E_{\max }$ is the maximum magnitude of the electric field in the cavity. The stored energy is basically proportional to the hollow volume of the cavity. Keeping the cavity-size constant, a cavity with mushroom-shaped cylinders has larger hollow volume compared to the cavity with uniform metallic cylinders, and generally has better power handling capability [20]. The power handling capability for the proposed filter with mushroom-shaped cylinders was $\zeta \approx 9.8 e^{-19}$, which was larger than that of the cavity filter with uniform metallic cylinders for keeping the same passband center frequency.

\subsection{Filter Realization}

The proposed quadruple-mode resonator was utilized to realize the wideband bandpass filter after achieving the appropriate dimensions for all the parameters. Optimized dimensions of the filter were $a=50, b=21$, ofst $=7.5, l_{p r b}=19.3, h_{c y l}=11, r_{c y l}=4.8, r_{c a p}=6$ (all in mm). The simulated results show that the stopband rejection level was better than $30 \mathrm{~dB}$ on both sides of the passband, below $2 \mathrm{GHz}$ on the lower side, whereas it was from $3.8 \mathrm{GHz}$ to $6.8 \mathrm{GHz}$ on upper side of the passband with a return loss that was also better than $30 \mathrm{~dB}$, as shown in Figure 8. The simulated insertion loss of the filter observed was less than $0.03 \mathrm{~dB}$.

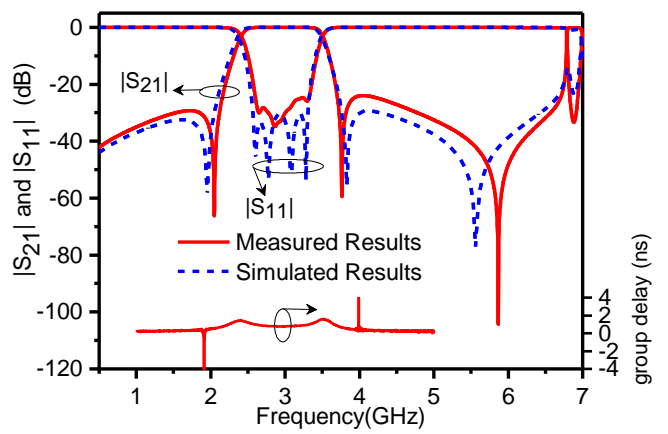

Figure 8. Simulated results (dotted) and measured results (solid) of reflection and transmission coefficients of the quadruple-mode filter with measured group delay.

\section{Experimental Results and Discussions}

The filter was designed at $2.93 \mathrm{GHz}$ with $38 \%$ wide fractional bandwidth. Figure 8 shows that the simulated and measured S-parameters of the filter agreed well with a small deviation probably caused by loss of the aluminum material used and tolerance in the fabrication of the cavity, cylinders, and probes. It was noticed that the tips of the input/output probes were slightly bent away from the 
mushroom-shaped cylinders, which was believed to be the main cause for the small deviation between simulated and measured results. The deviation could be minimized by selecting materials with low loss and improving the fabrication process, especially the probes. The measured insertion loss at the center frequency was $0.06 \mathrm{~dB}$, and the measured return loss for the wide passband was better than $25 \mathrm{~dB}$. The measured group delay showed considerable linearity along the wider passband of the filter. A prototype filter was fabricated using aluminum to validate the simulated results of the filter, as clearly photographed in Figure 9.

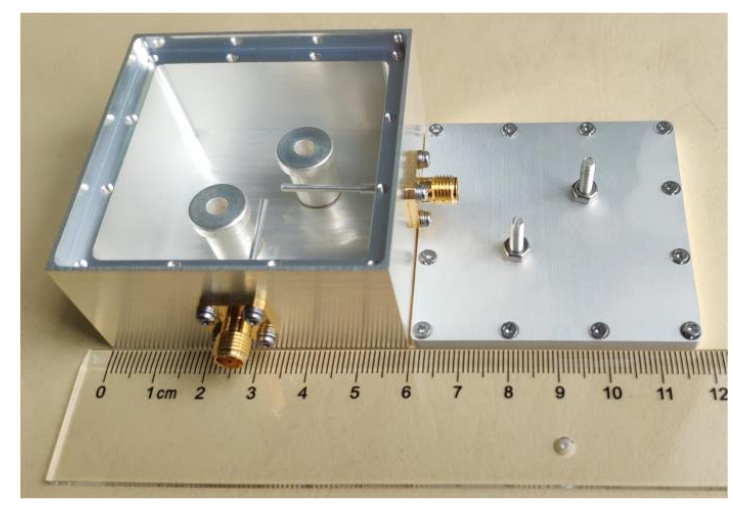

Figure 9. Fabricated quadruple-mode filter.

Table 1 presents the comparison of this filter with other similar cavity filters reported in recent past. The proposed filter outperformed the other works in the field of out-of-band rejection, fractional bandwidth, overall size, and insertion loss.

Table 1. Comparison among similar cavity filters.

\begin{tabular}{ccccccc}
\hline Ref. & Modes/Order & Cavity Size & $\begin{array}{c}\text { Centre Freq. } \\
\text { (GHz) }\end{array}$ & FBW (\%) & $\begin{array}{c}\text { Out-of-band } \\
\text { Rejection }\end{array}$ & $\begin{array}{c}\text { Measured } \\
\text { Insertion } \\
\text { Loss (dB) }\end{array}$ \\
\hline$[15]$ & $3 / 6$ th & $2^{*}\left(0.4 \lambda_{o}^{*} 0.4 \lambda_{o}^{*} 0.4 \lambda_{o}\right)$ & 2.10 & 2.1 & $1.63 f_{o},>60 \mathrm{~dB}$ & $<0.6$ \\
{$[18]$} & $4 / 8$ th & $2^{*}\left(0.18 \lambda_{o}^{*} 0.13 \lambda_{o}^{*} 0.18 \lambda_{o}\right)$ & 2.55 & 3.9 & $3 f_{o},>60 \mathrm{~dB}$ & $<0.77$ \\
{$[19]$} & $3 / 3 \mathrm{rd}$ & $\Phi 0.5 \lambda_{o}^{*} 0.5 \lambda_{o}$ & 3.2 & 30 & $1.5 f_{o},>20 \mathrm{~dB}$ & $<0.5$ \\
{$[19]$} & $4 / 4$ th & $\Phi 0.5 \lambda_{o}^{*} 0.5 \lambda_{o}$ & 3.2 & 31 & $1.4 f_{o},>20 \mathrm{~dB}$ & $<0.5$ \\
{$[20]$} & $3 / 3 \mathrm{rd}$ & $0.41 \lambda_{o}^{*} 0.2 \lambda_{o}^{*} 0.41 \lambda_{o}$ & 2.4 & 58 & $2 f_{o},>20 \mathrm{~dB}$ & $<0.1$ \\
{$[21]$} & $3 / 3 \mathrm{rd}$ & $1 / 2{ }^{*} 0.43 \lambda_{o}^{*} 0.5 \lambda_{o}^{*} 0.5 \lambda_{o}$ & 2.94 & 22 & $1.27 f_{o},>10 \mathrm{~dB}$ & $<0.1$ \\
This work & $4 / 4$ th & $0.5 \lambda_{o}^{*} 0.2 \lambda_{o}^{*} 0.5 \lambda_{o}$ & 2.93 & 38 & $2.32 f_{o},>30 \mathrm{~dB}$ & $<0.06$ \\
\hline
\end{tabular}

\section{Conclusions}

In this paper, a quadruple-mode wideband bandpass filter employing a rectangular waveguide cavity was presented. Two mushroom-shaped perturbation cylinders along with two perpendicularly inserted coaxial probes were used to excite the four resonant modes. The employment of mushroom-shaped cylinders resulted in a significant reduction of the cavity size. Height of the cavity and shape of the perturbation cylinders were exploited to improve the out-of-band rejection, selectivity, and miniaturization. A filter prototype was fabricated, and its performance was measured to validate the design. The measured results were in accordance with the simulated ones. The measured results of the filter showed low insertion loss $(<0.06 \mathrm{~dB})$, good return loss $(>25 \mathrm{~dB})$, wider fractional bandwidth (38\%), and improved out-of-band rejection $\left(2.32 f_{o},>30 \mathrm{~dB}\right)$.

Author Contributions: M.H. proposed, designed and wrote the paper; G.X. supervised the experiments and write-up of the paper; A.I.N., L.Q., and T.H. fabricated the filter and helped in carrying out experiments.

Funding: This work is partly supported by the National Natural Science Foundation of China (Granted: 61771304).

Conflicts of Interest: The authors declare no conflict of interest. 


\section{References}

1. Williams, A.E. A Four-Cavity Elliptic Waveguide Filter. IEEE Trans. Microw. Theory Tech. 1970, 18, 1109-1114. [CrossRef]

2. Atia, A.E.; Williams, A.E. Narrow-Bandpass Waveguide Filters. IEEE Trans. Microw. Theory Tech. 1972, 20, 258-265. [CrossRef]

3. Atia, A.E.; Williams, A.E.; Newcomb, R.W. Narrow-Band Multiple-Coupled Cavity Synthesis. IEEE Trans. Circ. Syst. 1974, 21, 649-655. [CrossRef]

4. Wang, Y.; Yu, M. True inline cross-coupled coaxial cavity filters. IEEE Trans. Microw. Theory Tech. 2009, 57, 2958-2965. [CrossRef]

5. Zhang, Q.; Lu, Y. Dimensional Synthesis of Symmetric Wideband Waveguide Cross-Coupled Filters without Global Full-Wave Optimization. IEEE Trans. Microw. Theory Tech. 2010, 58, 3742-3748. [CrossRef]

6. Zhu, L.; Sun, S.; Menzel, W. Ultra-Wideband (UWB) bandpass filters using multiple mode resonator. IEEE Microw. Wirel. Compon. Lett. 2005, 15, 796-798. [CrossRef]

7. Wang, H.; Zhu, L.; Menzel, W. Ultra-wideband bandpass filter with hybrid microstrip/CPW structure. IEEE Microw. Wirel. Compon. Lett. 2005, 15, 844-846. [CrossRef]

8. Liu, Z.; Xiao, G.; Zhu, L. A Novel Method to Design Triple-Mode SIW Filter Based on the Complementary Split Ring Resonators (CSRRs). IEEE MTT-S Int. Microw. Symp. Dig. 2016, 4-6. [CrossRef]

9. Liu, Z.; Xiao, G.; Zhu, L. Triple-Mode Bandpass Filters on CSRR-Loaded Substrate Integrated Waveguide Cavities. IEEE Trans. Compon. Packag. Manuf. Technol. 2016, 6, 1101-1107. [CrossRef]

10. Hameed, M.; Xiao, G.; Qiu, L.; Xiong, C.; Hameed, T. Multiple-ModeWideband Bandpass Filter Using Split Ring Resonators in a Rectangular Waveguide Cavity. Electronics 2018, 7, 356. [CrossRef]

11. Lin, W.G. Microwave Filters Employing a Single Cavity Excited in More than One Mode. J. Appl. Phys. 1951, 22, 989-1001. [CrossRef]

12. Fiedziuszko, S.J. Dual-mode dielectric resonator loaded cavity filters. IEEE Trans. Microw. Theory Tech. 1982, 30, 1311-1316. [CrossRef]

13. Memarian, M.; Mansour, R.R. Quad-mode and dual-mode dielectric resonator filters. IEEE Trans. Microw. Theory Tech. 2009, 57, 3418-3426. [CrossRef]

14. Tang, W.-C.; Chaudhuri, S.K. A true elliptic-function filter using triple-mode degenerate cavities. IEEE Trans. Microw. Theory Tech. 1984, 32, 1449-1454. [CrossRef]

15. Salehi, H.; Bernhardt, T.; Lukkarila, T.; Amir, S. Analysis, design and applications of the triple-mode conductor-loaded cavity filter. IET Microw. Antennas Propag. 2011, 5, 1136-1142. [CrossRef]

16. Bonetti, R.R.; Williams, A.E. Application of Dual TM Modes to Triple-and Quadruple-Mode Filters. IEEE Trans. Microw. Theory Tech. 1987, 35, 1143-1149. [CrossRef]

17. Liang, X.-P.; Zaki, K.A.; Atia, A.E. Dual Mode Coupling by Square Corner Cut in Resonators and Filters. IEEE Trans. Microw. Theory Tech. 1992, 40, 2294-2302. [CrossRef]

18. Wang, X.; Jang, G.; Lee, B.; Park, N. Compact quad-mode bandpass filter using modified coaxial cavity resonator with improved $Q$-factor. IEEE Trans. Microw. Theory Tech. 2015, 63, 965-975. [CrossRef]

19. Wong, S.-W.; Feng, S.-F.; Zhu, L. Triple- and quadruple-mode wideband bandpass. IEEE Trans. Microw. Theory Tech. 2015, 63, 3416-3424. [CrossRef]

20. Hameed, M.; Xiao, G.; Qiu, L. Triple-mode wideband bandpass filter using simple perturbation in metallic-loaded rectangular waveguide cavity. Electromagnetics 2018, 38, 303-316. [CrossRef]

21. Hameed, M.; Xiao, G.; Xiong, C. Triple-mode wideband bandpass filter using triangular waveguide cavity. In Proceedings of the IEEE MTT-S IWS, Chengdu, China, 6-10 May 2018; pp. 1-3.

22. Cameron, R.J. Advanced coupling matrix synthesis techniques for microwave filters. IEEE Trans. Microw. Theory Tech. 2003, 51, 1-10. [CrossRef]

23. Meng, M.; Wu, K.-L. An analytical approach to computer-aided diagnosis and tuning of lossy microwave coupled resonator filters. IEEE Trans. Microw. Theory Tech. 2009, 57, 3188-3195. [CrossRef]

(C) 2019 by the authors. Licensee MDPI, Basel, Switzerland. This article is an open access article distributed under the terms and conditions of the Creative Commons Attribution (CC BY) license (http://creativecommons.org/licenses/by/4.0/). 